\title{
Community-acquired pneumonia distinguished from influenza infection based on clinical signs and symptoms during a novel (swine) influenza A/H1N1 pandemic
}

\author{
*Masanori Nakanishia, Yishimasa Yoshidaa, Nobuya Takedab, Hirohumi Hiranab, \\ Takahiro Horitab, Kazuhiko Shimizu, Kazuyuki Hiratani ${ }^{b}$, Shigeo Toyodab, \\ Takayuki Matsumura ${ }^{b}$, Eiji Shinno ${ }^{b}$, Akihiro Hutamurab ${ }^{b}$ Masanari Ota ${ }^{b}$, Toshoki Natazuka ${ }^{b}$ \\ a Department of Respiratory Medicine, Shinseikai Toyama Hospital, Imizu, Japan
${ }^{\mathrm{b}}$ Department of Internal Medicine, Shinseikai Toyama Hospital, Imizu, Japan
}

Received 29th January 2011; revised 2nd April 2011; accepted 2nd May 2011; online 2nd August 2011

\begin{abstract}
Aims: The numbers of patients with influenza-like illnesses increase during influenza outbreaks. A study was undertaken to distinguish community-acquired pneumonia (CAP) from influenza based on clinical signs and symptoms.

Methods: This retrospective study investigated patients with positive results in the rapid influenza antigen test and those diagnosed with CAP during an influenza A/H1N1 pandemic. Significant factors for predicting risk for CAP within 48 hrs from onset and at diagnosis were selected by multiple regression analysis.

Results: Within 48 hrs of onset and at diagnosis, age, sputum and coarse crackles significantly increased the risk of CAP whereas sick contact, sore throat, and rhinorrhoea significantly decreased the risk of CAP. Duration of illness, sputum, dyspnoea, chest pain, and coarse crackles also significantly increased the risk of CAP at diagnosis.

Conclusions: CAP differed somewhat from influenza even within 48 hrs of onset and the differences became even more evident thereafter.

(C) 2011 Primary Care Respiratory Society UK. All rights reserved.

M Nakanishi et al. Prim Care Respir J 2011; 20(4): 421-426

http://dx.doi.org/pcrj.2011.00067
\end{abstract}

Keywords primary care, pulmonary diseases, differential diagnosis, signs, symptoms, community-acquired pneumonia, influenza $\mathrm{A} / \mathrm{H} 1 \mathrm{~N} 1$

\section{See linked editorial by Lim on pg 353}

\section{Introduction}

The numbers of patients who become ill with viral influenza increase during influenza epidemics. However, many other diseases also present as influenza-like illnesses (ILI), including community-acquired pneumonia (CAP). To distinguish influenza from ILI is important, yet the sensitivity of the rapid influenza antigen test (RIAT) is low ${ }^{1,2}$ and diagnosing influenza based on clinical signs and symptoms is difficult. ${ }^{3-5}$ Nevertheless, primary care physicians in this setting without a gold standard for diagnosing influenza infection must reach a decision as to whether to treat the source of the infection with antiviral agents or antibiotics, or to treat only the symptoms.

Many patients present with CAP caused by other pathogens or influenza or with co-infection during influenza epidemics. ${ }^{6-8}$ Misdiagnosed or overlooked conditions such as pneumonia that actually require antibiotic treatment could negatively impact the prognosis. ${ }^{910}$ In addition, although most influenza is self-limiting and the incidence of pneumonia presenting with influenza is low - even in the general population infected with novel influenza $\mathrm{A} / \mathrm{H} 1 \mathrm{~N} 1^{11-14}$ - antibiotics are recommended for treating patients with influenza-associated pneumonia because the mortality rate is higher for influenza-associated pneumonia with bacterial infection. ${ }^{7.8}$ Patients with CAP should therefore be differentiated from those with ILI.

\footnotetext{
* Corresponding author: Dr Masanori Nakanishi, Department of Respiratory Medicine, Shinseikai Toyama Hospital, 89-10, Shimowaka, Imizu City, Toyama 939-0243, Japan, Imizu 939-0243, Japan. Tel: +81 766522156 Fax: +81 766522197 E-mail: mnakanishi@nifty.ne.jp
} 
However, to diagnose CAP based on clinical signs and symptoms is also difficult. ${ }^{15}$ Patients with ILI during epidemics who have cough and fever are likely to have influenza, ${ }^{3-5}$ yet these symptoms are also the main symptoms of $C A P^{15,16}$ and abnormal chest findings have a high specificity but a low sensitivity for diagnosing pneumonia. ${ }^{17,18}$ In addition, although an early diagnosis of influenza is necessary because neuraminidase inhibitors should be prescribed within $48 \mathrm{hrs},{ }^{19}$ to differentiate influenza from CAP early is quite difficult.

A novel influenza A/H1N1 strain emerged in 2009 and was declared a pandemic by the World Health Organization. A retrospective study was undertaken of the diagnostic yield of signs and symptoms in distinguishing CAP from influenza infection during this pandemic. In addition, we investigated whether CAP could be distinguished from influenza within 48 hrs of onset in the primary care setting.

\section{Methods}

\section{Setting}

This study was undertaken at a single institution in Imizu City (Toyama Prefecture, Japan) with a population of 100,000 . Of the approximately 200 persons per day presenting at our internal medicine department or at the emergency department of the outpatient clinic, about 10-20 are new patients with acute respiratory symptoms. However, during the influenza $\mathrm{A} / \mathrm{H} 1 \mathrm{~N} 1$ pandemic, about 30-60 new patients per day presented at these two departments with acute respiratory symptoms. Most of these patients presented directly at our institution without attending other GPs or being visited by home doctors. These patients were therefore considered to be in a primary care setting.

\section{Patients}

During the novel influenza A/H1N1 pandemic, 3,464 patients with ILI were tested at our institution between September 2009 and February 2010 with the RIAT. However, not all patients with high pretest probabilities were tested - for example, those from clusters such as households, offices, or schools - because the sensitivity of the RIAT is low.

Patients with positive RIAT results were regarded as having an influenza infection because the specificity of the test - even for influenza A/H1N1 - is high, ${ }^{20-22}$ and modalities such as real-time reverse transcription polymerase chain reaction testing (rRT-PCR) were not available in our primary care setting. The diagnosis of CAP was based on new infiltrates on chest x-rays and a clinical course that was cured by antibiotics. Two expert respiratory specialists who were independently blinded to the clinical information and diagnosis interpreted the chest $x$-rays. Disagreements were resolved by consensus. Patients with positive RIAT results were retrospectively compared with those who had CAP.

The following types of patients were excluded: those diagnosed with other pulmonary infectious diseases during the clinical course; those aged $<15$ or $>75$ years; those with dementia or cerebral vascular diseases; and those with other confirmed types of acute infectious diseases, chronic respiratory or malignant diseases, or immunodeficiency. Patients referred to our institution by any other physician were also excluded. All the data and patients described here are novel and were not part of our previous study. ${ }^{18}$ Our institutional review board approved the study. Written informed consent from each patient was not required as this study was retrospective and involved only patient records and images.

\section{Aetiology}

Sputum obtained from patients with CAP by either spontaneous expectoration or with the help of nebulised saline was cultured to determine bacteria and acid-fast bacilli. Venous blood samples were tested for Mycoplasma pneumoniae during the acute phase and 1-2 weeks after the first hospital visit. A more than fourfold increase in antibodies was deemed positive. Urinary Streptococcus pneumoniae antigen was tested in urine samples. The aetiologies of other conditions including Coxiella burnetii, Legionella pneumophila, and Chlamydia pneumoniae were examined in patients who were suspected of having these diseases. Blood was cultured only when sepsis was suspected. Serological samples were not tested for viruses.

\section{Analysis of data}

Data were extracted from the clinical records for each patient. Clinical signs and symptoms included age, temperature, cough, sputum, sore throat, rhinorrhoea, headache, myalgia, malaise, arthralgia, appetite loss, dyspnoea, chest pain, duration of illness, lymphadenopathy, swollen pharynges, coarse crackles, wheezing, heart rate, and systolic blood pressure at diagnosis. Extensive sick contact in clusters such as households, schools, or offices was also recorded. Significant factors for predicting the risk of CAP at diagnosis were selected by multiple regression analysis.

Patients who were tested by RIAT at our institution within 48 hrs of disease onset were selected from among those described above to evaluate the diagnostic yields of signs and symptoms within this time frame. Clinical signs and symptoms at the first hospital visit within 48 hrs of disease onset were also extracted from the clinical records of the patients and significant factors for predicting the risk for CAP were selected by multiple regression analysis.

\section{Statistical analysis}

Data were analysed using SPSS Version 11.0 for Windows. Clinical factors including signs and symptoms significantly associated with the risk of CAP were selected by multiple logistic regression analysis. The 95\% confidence intervals (95\% CI) were calculated for each comparison. All tests of significance were two-tailed and a p value of $<0.05$ was considered significant.

\section{Results}

Figures 1 and 2 show the numbers of patients in each category. Of 3,464 patients who were tested by RIAT during the study period, 718 were positive and 68 had CAP (Figure 1). 
Figure 1. Numbers of patients in each category among 3,464 tested by rapid influenza antigen test (RIAT)

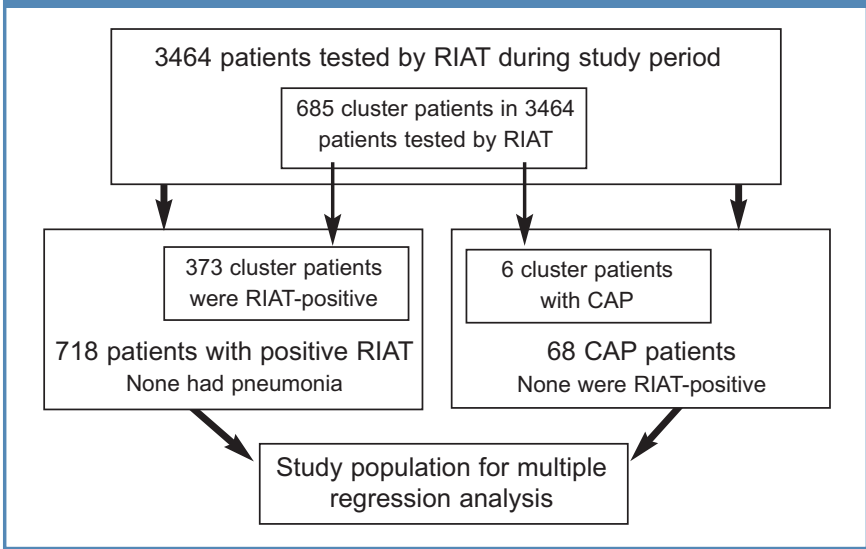

Chest $x$-rays in 72 of the 718 patients with positive RIAT findings confirmed that none of them had pneumonia. Therefore, our cohort did not contain any patients with CAP who were also RIAT-positive. None of the patients with influenza were admitted to our hospital. Fifteen of the 68 patients with CAP were admitted to our hospital and all those with CAP improved with antibiotic therapy. Table 1 summarises the aetiologies of CAP. Among 3,464 patients who were tested by RIAT, 685 were from clusters such as households, offices, or schools. Of these 685 patients, 373 (54\%) were RIAT-positive and six (0.9\%) had CAP.

Of the 3,464 patients who were tested by RIAT, 2,617 attended our institution within 48 hrs of onset. Of these 2,617 patients, 586 were RIAT-positive and 31 had CAP (Figure 2). None of these patients had both CAP and RIAT positivity. Of the 586 RIAT-positive patients, 528 were positive at the first testing and 58 were positive at a second or third testing. Among the 31 patients with CAP, only 16 were diagnosed at the first visit within 48 hrs of onset, whereas 11 , three and one patients were diagnosed at the second, third and fourth tests, respectively. Among the 2,617 patients who presented at our institution within 48 hrs of onset and who were tested by RIAT, 641 were from clusters, of whom 316 (49\%) were RIAT-positive and five $(0.8 \%)$ had CAP.

The results of multiple regression analyses of the clinical signs and symptoms at the first presentation within 48 hrs of onset for predicting a risk of CAP are presented in Table 2. Age, sputum and coarse crackles significantly increased the risk of CAP. Sick contact, sore throat, and rhinorrhoea significantly decreased the risk of CAP.

The results of multiple regression analyses of the clinical signs and symptoms at diagnosis for predicting a risk of CAP in all patients with positive RIAT results and in all patients with CAP are presented in Table 3. Age, duration of illness, sputum, dyspnoea, chest pain, and coarse crackles significantly increased the risk of CAP, whereas sick contact, sore throat, and rhinorrhoea significantly decreased the risk of CAP.
Figure 2. Numbers of patients in each category among 2,617 attending our institution within 48 hrs of onset and tested by rapid influenza antigen test (RIAT)

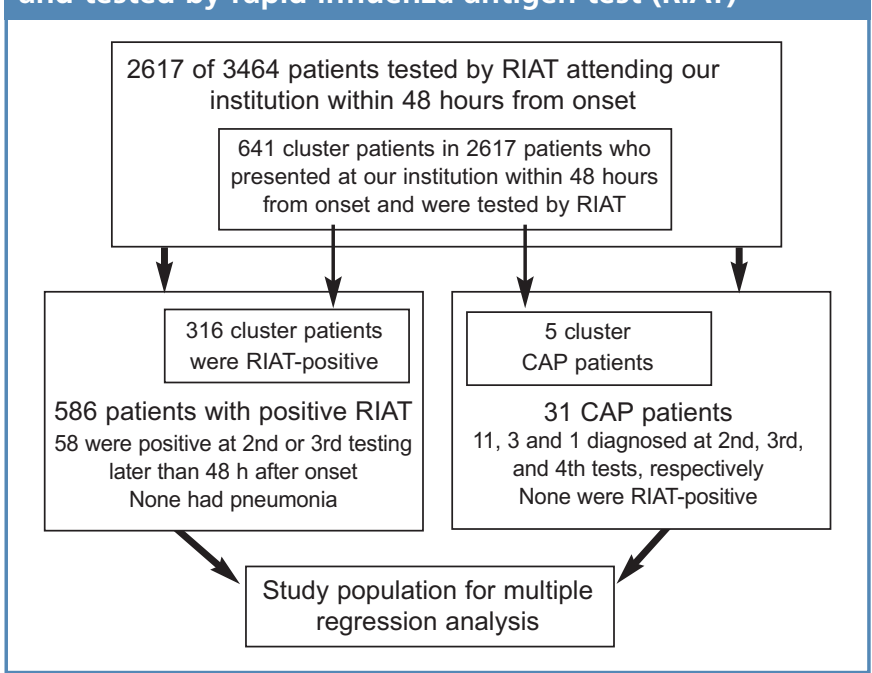

Table 1. Bacterial pathogens in patients with pneumonia

\begin{tabular}{ll} 
Aetiology & $\mathrm{n}(\%)$ \\
\hline Streptococcus pneumoniae & $17(25)$ \\
Mycoplasma pneumoniae & $12(17.6)$ \\
Haemophilus influenzae & $7(10.3)$ \\
Staphylococcus aureus & $3(4.4)$ \\
Klebsiella pneumoniae & $3(4.4)$ \\
Moraxella catarrhalis & $2(2.9)$ \\
Unknown & $24(35.2)$ \\
Total & 68
\end{tabular}

\section{Discussion}

The findings of this study show that CAP is a widespread disease that presented as ILI during the 2009 pandemic. Influenza and CAP are not easy to distinguish based on signs and symptoms, as only about half of patients with CAP could be diagnosed at first hospital presentation within 48 hrs of disease onset. However, CAP and influenza infection differed somewhat. More advanced age and the absence of a sore throat or rhinorrhoea significantly increased the risk of CAP, even within 48 hrs from onset. A few days after onset, sputum also significantly increased the risk of CAP, and relatively specific findings for pneumonia such as dyspnoea or chest pain or coarse crackles were likely to be evident in patients with CAP. Findings specific to CAP would become more obvious over time because pneumonia is most likely to deteriorate without antibiotic treatment., ${ }^{9,10,18}$

The definition of sick contact in this study is extensive contact derived from households, offices, or schools. Almost half of our cohort from such environments had positive RIAT results, although not all of them were necessarily tested because the sensitivity of RIAT ranges from $47 \%$ to $64 \%$ during influenza A/H1N1 pandemics ${ }^{20-22}$ and almost $70 \%$ of patients with ILI and extensive sick contact are positive in rRT-PCR tests for H1N1. ${ }^{14}$ 
M Nakanishi et al.

\begin{tabular}{|c|c|c|c|c|}
\hline & Positive RIAT $(n=586)$ & CAP $(n=31)$ & OR $(95 \% \mathrm{Cl})$ & $\mathrm{p}$ value \\
\hline Age (yrs) & $28.2 \pm 10.8$ & $49.0 \pm 18.6$ & $1.338(1.166$ to 1.961$)$ & 0.0001 \\
\hline Sex (female) & $59 \%$ & $47 \%$ & $0.877(0.453$ to 1.877$)$ & 0.616 \\
\hline Sick contact* & $54 \%$ & $16 \%$ & $0.072(0.019$ to 0.292$)$ & 0.0001 \\
\hline Temperature & $38.3 \pm 0.7$ & $38.3 \pm 0.8$ & $1.236(0.581$ to 2.629$)$ & 0.582 \\
\hline Cough & $83 \%$ & $78 \%$ & $0.689(0.207$ to 2.290$)$ & 0.543 \\
\hline Sputum & $21 \%$ & $41 \%$ & 4.041 (1.315 to 12.419$)$ & 0.006 \\
\hline Sore throat & $51 \%$ & $21 \%$ & $0.341(0.118$ to 0.988$)$ & 0.048 \\
\hline Rhinorrhoea & $50 \%$ & $20 \%$ & $0.315(0.103$ to 0.962$)$ & 0.045 \\
\hline Dyspnoea & $2 \%$ & $19 \%$ & $4.885(0.718$ to 33.212$)$ & 0.105 \\
\hline Chest pain & $2 \%$ & $14 \%$ & $5.263(0.804$ to 34.446$)$ & 0.083 \\
\hline Headache & $35 \%$ & $27 \%$ & $1.600(0.545$ to 4.701$)$ & 0.393 \\
\hline Fatigue & $37 \%$ & $46 \%$ & $1.951(0.652$ to 5.838$)$ & 0.232 \\
\hline Myalgia & $24 \%$ & $22 \%$ & $0.543(0.164$ to 1.800$)$ & 0.318 \\
\hline Arthralgia & $25 \%$ & $33 \%$ & 0.656 (0.184 to 2.335) & 0.515 \\
\hline Coarse crackles & $1 \%$ & $28 \%$ & 17.306 (3.495 to 85.708$)$ & 0.0001 \\
\hline Wheeze or rhonchi & $1 \%$ & $1 \%$ & $0.899(0.112$ to 97.155$)$ & 0.900 \\
\hline Neck lymphadenopathy & $2 \%$ & $2 \%$ & $0.815(0.136$ to 85.775$)$ & 0.887 \\
\hline Tonsillitis & $6 \%$ & $2 \%$ & $0.654(0.209$ to 56.355$)$ & 0.696 \\
\hline
\end{tabular}

Data are presented as numbers (\%) or means \pm standard deviation.

*Sick contact in this study is defined as extensive contact derived from households, offices, or schools.

$\mathrm{CAP}=$ community acquired pneumonia; $\mathrm{Cl}=$ confidence interval; $\mathrm{OR}=$ odds ratio; $\mathrm{RIAT}=$ rapid influenza antigen test.

Table 3. Findings of multiple regression analysis of clinical signs and symptoms at diagnosis for predicting risk of CAP in all RIAT-positive patients and in all those with CAP

\begin{tabular}{|c|c|c|c|c|}
\hline & Positive RIAT $(n=718)$ & CAP $(n=68)$ & OR $(95 \% \mathrm{Cl})$ & $\mathrm{p}$ value \\
\hline Age (yrs) & $28.9 \pm 11.2$ & $53.5 \pm 20.6$ & $1.919(1.891$ to 1.947$)$ & 0.0001 \\
\hline Sex (female) & $58 \%$ & $48 \%$ & $0.827(0.239$ to 12.255$)$ & 0.951 \\
\hline Sick contact* & $52 \%$ & $9 \%$ & 0.145 (0.0297 to 0.267$)$ & 0.0001 \\
\hline Duration of illness (days) & $2.0 \pm 1.1$ & $5.0 \pm 3.2$ & 1.515 (1.401 to 1.662$)$ & 0.001 \\
\hline Temperature & $38.3 \pm 0.7$ & $38.4 \pm 0.7$ & 1.341 (1.170 to 1.686$)$ & 0.766 \\
\hline Cough & $86 \%$ & $94 \%$ & 5.026 (0.513 to 49.249$)$ & 0.166 \\
\hline Sputum & $29 \%$ & $65 \%$ & $3.102(1.247$ to 7.720$)$ & 0.025 \\
\hline Sore throat & $54 \%$ & $24 \%$ & $0.245(0.076$ to 0.785$)$ & 0.028 \\
\hline Rhinorrhoea & $53 \%$ & $20 \%$ & $0.357(0.114$ to 0.651$)$ & 0.048 \\
\hline Dyspnoea & $3 \%$ & $25 \%$ & 4.974 (1.271 to 19.470$)$ & 0.021 \\
\hline Chest pain & $4 \%$ & $14 \%$ & $5.348(1.435$ to 19.929$)$ & 0.012 \\
\hline Headache & $29 \%$ & $22 \%$ & 0.981 (0.353 to 2.730$)$ & 0.971 \\
\hline Fatigue & $41 \%$ & $56 \%$ & $1.019(0.377$ to 2.758$)$ & 0.970 \\
\hline Myalgia & $21 \%$ & $24 \%$ & $0.473(0.156$ to 1.434$)$ & 0.186 \\
\hline Arthralgia & $32 \%$ & $28 \%$ & $0.675(0.256$ to 1.781$)$ & 0.427 \\
\hline Coarse crackles & $1 \%$ & $38 \%$ & 39.294 (7.176 to 215.156) & 0.0001 \\
\hline Wheeze or rhonchi & $1 \%$ & $2 \%$ & $1.886(0.177$ to 25.135$)$ & 0.865 \\
\hline Neck lymphadenopathy & $2 \%$ & $3 \%$ & $1.599(0.147$ to 17.434$)$ & 0.700 \\
\hline Tonsillitis & $7 \%$ & $2 \%$ & $5.106(0.279$ to 93.504$)$ & 0.272 \\
\hline
\end{tabular}


Most patients with a high pretest probability of influenza infection were from clusters, and typical symptoms would indicate a provisional diagnosis of influenza infection in the primary care setting. However, this did not mean that all patients from clusters would always have influenza. In fact, about $1 \%$ of such patients had CAP, which indicates a need for caution when considering the diagnosis of patients presenting with ILI. Even cluster patients should undergo the RIAT when they are at high risk or have co-morbidities such as pneumonia.

Because the transmission rate of influenza is higher, sick contact should be the most significant factor for influenza infection. ${ }^{13,14}$ More advanced age is a significant factor for CAP because the transmission rate in children and young adults during the H1N1 pandemic is higher ${ }^{13,14}$ and the incidence of pneumonia is higher among the elderly. ${ }^{6}$ On the other hand, cough and fever are the main symptoms of both influenza infection and CAP, which causes difficulties in distinguishing between them. ${ }^{45,15,16}$ Because sputum tends to have a positive predictive value and sore throat and rhinorrhoea tend to have a negative predictive value for $C A P_{1}{ }^{15}$ these factors could be useful for differentiation. However, the differences between each of these factors were small within $48 \mathrm{hrs}$ from onset. The notable findings of the present study were that differences between CAP and influenza became clearer, and more characteristic findings for CAP including dyspnoea, chest pain and coarse crackles became significant risk factors for CAP over time. This could be because CAP deteriorates without antibiotic therapy and symptoms specific to pneumonia start to develop, whereas influenza usually improves after several days.

Differences in the signs and symptoms of influenza and CAP have not been investigated in detail, although some studies have examined predictors for CAP during periods other than influenza outbreaks. ${ }^{15-18}$ This could be due to difficulties associated with ruling out influenza infection in patients with CAP in primary care because rRT-PCR testing is not available in many clinical settings. However, the significance of treating patients with ILI, most of which was self-limiting, was to decrease the likelihood of missing patients who require optimal therapy for non-self-limiting diseases such as CAP. This study was conducted to determine clinical symptoms and signs that can distinguish CAP from influenza.

The major limitations of this study are that it was retrospective and included a single institution without an adequate reference standard for diagnosing influenza infection. Many patients with influenza should have false-negative RIAT results, and the RIAT was applied based on corresponding physicians' decisions for patients from clusters because the number of patients with false-negative results in diagnostic tests with low sensitivity increases in such populations. Therefore, this study did not include all patients with influenza at our institution during the pandemic. However, because the test is highly specific even for influenza $\mathrm{A} / \mathrm{H} 1 \mathrm{~N} 1,{ }^{20-22}$ nearly all patients with positive results were regarded as having influenza infection. Even patients from clusters should be examined by the RIAT when clinical signs or symptoms are atypical or when a definite diagnosis is necessary due to high risk.

None of our patients with positive RIAT results had CAP. This might be due to false-negative RIAT results, to missing those with mild influenza-associated pneumonia, or to patients with severe influenza attending other institutions. However, the incidence of pneumonia with influenza A/H1N1 infection is low in the general population. ${ }^{11-14}$ In addition, these patients with CAP were representative because the rate of pathogen detection and the bacterial aetiologies were similar to those in previous studies. ${ }^{23,24}$ Therefore, most of the CAP in this study was probably caused by bacterial infection in the absence of influenza. This study could identify significant factors that distinguish CAP from influenza during the A/H1N1 pandemic, and to differentiate CAP with or without influenza is significant because pneumonia requires optimal treatment. A study using an optimal reference standard for influenza A/H1N1 and chest radiography for diagnosing pneumonia in all patients with ILI might generate clearer results.

On the other hand, many patients with influenza A/H1N1 infection who are hospitalised or admitted to emergency departments have pneumonia. ${ }^{25,26}$ Primary care physicians should be aware of such conditions because influenza-associated pneumonia can rapidly progress to respiratory distress and extensive pulmonary involvement. ${ }^{27}$ This study could not identify differences between CAP with and without influenza or between influenza infection with and without CAP.

The transmission rate in children and young adults during the H1N1 pandemic was higher, ${ }^{13,14}$ which is a different course from the usual seasonal influenza. However, because the clinical features of A/H1N1 and seasonal influenza are almost identical, ${ }^{3-}$ 5,11-14 the results of this study could also be useful during seasonal influenza epidemics. This study excluded older patients, those with dementia, cerebral vascular diseases, immunodeficiency, or malignant and chronic respiratory diseases because the signs, symptoms, and clinical course of influenza infection in such patients and in normally healthy adults might differ. Most of our study population included otherwise healthy adults who comprise the majority of patients with ILI seen in general practice. In addition, many other infectious diseases of the airway presented as ILI including the common cold, acute sinusitis, streptococcus pharyngitis and infectious mononucleosis. This study did not compare the features of influenza infection with these conditions, although to differentiate such diseases from influenza is also important. However, overlooking CAP could negatively impact the prognosis among the various types of ILI.

Many patients with ILI including CAP attend GPs or emergency departments during influenza epidemics. To distinguish CAP from influenza infection based on clinical signs and symptoms soon after onset is difficult. The findings of this study during the influenza A/H1N1 pandemic uncovered a few 
differences between CAP and influenza infection even within 48 hrs of onset, and even more differences became evident thereafter. More advanced age, absence of sick contact, duration of illness, sputum, absence of sore throat or rhinorrhoea, chest pain, dyspnoea, and coarse crackles were significant factors with which to predict CAP during the influenza A/H1N1 pandemic. Evaluating these factors is useful to select patients with suspected or probable CAP and to decrease the likelihood of missing CAP even during seasonal influenza epidemics.

\section{Handling editor}

Irem Patel

\section{Statistical review}

Gopal Netuveli

\section{Conflicts of interest}

The authors declare that they have no conflicts of interest in relation to this article.

\section{Contributorship}

All authors clinically treated corresponding patients in this study. Nakanishi and Yoshimasa Yoshida reviewed all chest X-rays and clinical records.

\section{Funding}

None.

\section{References}

1. Petric $\mathrm{M}, \mathrm{C}$ Comanor $\mathrm{L}$, Petti CA. Role of the laboratory in diagnosis of influenza during seasonal epidemics and potential pandemics. J Infect Dis 2006;194:598110. http://dx.doi.org/10.1086/507554

2. Ruest $A$, Michaud $S$, Deslandes $S$, Frost EH. Comparison of the Directigen flu $A+B$ test, the QuickVue influenza test, and clinical case definition to viral culture and reverse transcription-PCR for rapid diagnosis of influenza virus infection. I Clin Microbiol 2003;41:3487-93. http://dx.doi.org/10.1128/JCM.41.8.3487-8793.2003.

3. Call SA, Vollenweider MA, Hornung CA, Simel DL, MCKinney WP. Does this patient have influenza? JAMA 2005;23:987-97. http://dx.doi.org/10.1001/jama.293.8.987.

4. Monto AS, Gravenstein S, Elliott M, Colopy M, Schweinle J. Clinical signs and symptoms predicting influenza infection. Arch Intern Med 2000;160:3243-7.

5. Zambon M, Hays J, Webster A, Newman R, Keene O. Diagnosis of influenza in the community: relationship of clinical diagnosis to confirmed virological, serologic, or molecular detection of influenza. Arch Intern Med 2001;161:2116-22.

6. Foy HM, Cooney MK, Allan I, Kenny GE. Rates of pneumonia during influenza epidemics in Seattle, 1964 to 1975. JAMA 1979;241:253-8. http://dx.doi.org/10.1001/jama.1979.03290290021018.

7. von Baum H, Schweiger B, Welte T, et al; The Capnetz Study Group. How deadly is seasonal influenza associated pneumonia? The German Competence Network for Community-acquired pneumonia (CAPNETZ). Eur Respir J 2011;37:1151-7. http://dx.doi.org/10.1183/09031936.00037410.

8. Hageman JC, Uyeki TM, Francis JS, et al. Severe community-acquired pneumonia due to Staphylococcus aureus, 2003-04 influenza season. Emerg Infect Dis 2006;12:894-9.

9. Winchester CC, Macfarlane TV, Thomas M, Price D. Antibiotic prescribing and outcomes of lower respiratory tract infection in UK primary care. Chest 2009;135:1163-72. http://dx.doi.org/10.1378/chest.07-2940.

10. Petersen I, Johnson AM, Islam A, Duckworth G, Livermore DM, Hayward AC.
Protective effect of antibiotics against serious complications of common respiratory tract infections: retrospective cohort study with the UK General Practice Research Database. BMJ 2007;335:982. http://dx.doi.org/10.1136/bmj.39345.405243.BE.

11. Novel Swine-Origin Influenza A (H1N1) Virus Investigation Team, Dawood FS, Jain $S$, Finelli $L$, et al. Emergence of a novel swine-origin influenza A (H1N1) virus in humans. N Engl I Med 2009;360:2605-15.

12. Cao B, Li XW, Mao Y, et al; National Influenza A Pandemic (H1N1) 2009 Clinical Investigation Group of China. Clinical features of the initial cases of 2009 pandemic influenza A (H1N1) virus infection in China. N Engl J Med 2009:361:2507-17.

13. Centers for Disease Control and Prevention (CDC). Swine-origin influenza $A$ (H1N1) virus infections in a school - New York City, April 2009. MMWR Morb Mortal Wkly Rep 2009;58:470-2.

14. Crum-Cianflone NF, Blair PJ,et al. Clinical and epidemiologic characteristics of an outbreak of novel H1N1 (swine origin) influenza A virus among United States military beneficiaries. Clin Infect Dis 2009;49:1801-10. http://dx.doi.org/10.1086/648508.

15. Metlay JP, Kapoor WN, Fine MJ. Does this patient have community-acquired pneumonia? JAMA 1997;278:1440-5. http://dx.doi.org/10.1001/ jama.1997.03550170070035.

16. Gennis P, Gallagher J, Falvo C, Baker S, Than W. Clinical criteria for the detection of pneumonia in adults: guidelines for ordering chest roentgenograms in the emergency department. J Emerg Med 1989;7:263-8.

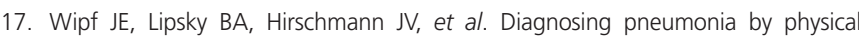
examination. Arch Intern Med 1999;159:1082-7.

18. Nakanishi M, Yoshida $Y$, Takeda $N$, et al. Significance of the progression of respiratory symptoms for predicting community-acquired pneumonia in general practice. Respirology 2010;15:969-74.

19. McNicholl IR, McNicholl JJ. Neuraminidase inhibitors: zanamivir and oseltamivir. Ann Pharmacother 2001;35:57-70. http://dx.doi.org/10.1345/aph.10118.

20. Centers for Disease Control and Prevention (CDC). Performance of rapid influenza diagnostic tests during two school outbreaks of 2009 pandemic influenza A (H1N1) virus infection - Connecticut, 2009. MMWR Morb Mortal Wkly Rep 2009;58:1029-32.

21. Vasoo S, Stevens J, Singh K. Rapid antigen tests for diagnosis of pandemic (Swine) influenza A/H1N1. Clin Infect Dis 2009;49:1090-3. http://dx.doi.org/10.1086/ 644743

22. Gordon A, Videa E, Saborío S, et al. Diagnostic accuracy of a rapid influenza test for pandemic influenza A H1N1. PLoS One 2010;5:e10364. http://dx.doi.org/ 10.1371/journal.pone.0010364.

23. Miyashita N, Fukano $\mathrm{H}$, Mouri $\mathrm{K}$, et al. Community-acquired pneumonia in Japan: a prospective ambulatory and hospitalized patient study. J Med Microbio/ 2005;54:395-400. http://dx.doi.org/10.1099/jmm.0.45920-0.

24. Woodhead M. Community-acquired pneumonia in Europe: causative pathogens and resistance patterns. Eur Respir J 2002;20(Suppl 36):2-27s. http://dx.doi.org/10.1183/09031936.02.00702002.

25. Jain S, Kamimoto L, Bramley AM, et al; 2009 Pandemic Influenza A (H1N1) Virus Hospitalizations Investigation Team. Hospitalized patients with 2009 H1N1 influenza in the United States, April-June 2009. N Engl J Med 2009;361(20):1935-44.

26. Di Giambenedetto S, Zileri Dal Verme L, Sali M, et al. Clinical presentation, microbiological features and correlates of disease severity of 2009 pandemic influenza A (H1N1) infection. Eur J Clin Microbiol Infect Dis 2011;30:541-9. http://dx.doi.org/10.1007/s10096-010-1116-7.

27. Rello J, Pop-Vicas A. Clinical review: primary influenza viral pneumonia. Crit Care 2009:13:235-41. http://dx.doi.org/10.1186/cc8183.

\section{Available online at http://www.thepcrj.org}

